\title{
PREDICTIVE CONTROL WITH ROBUST \\ DEAD-TIME COMPENSATION: APPLICATION TO DRUG DOSING DURING ANESTHESIA
}

\author{
Bismark Claure Torrico ${ }^{*}$ Robin De Keyser ** \\ Clara Ionescu ${ }^{* *}$ Julio E. Normey-Rico* \\ * Depto. de Automação e Sistemas, Universidade Federal \\ de Santa Catarina, Cx.P. 476, 88040-900, Florianopolis, \\ SC Brazil. \\ ** EeSA-Department of Electrical energy, Systems and \\ Automation, Ghent University Technologiepark 913, 9052 \\ Gent Belgium.
}

\begin{abstract}
This paper presents the application of predictive control to drug dosing during anesthesia in patients undergoing surgery. A single-input (Propofol) singleoutput (Bispectral index) model of the patient has been assumed which includes the variable dead time caused by the measurement of the Bispectral index. A set of 12 patient models were studied. Inter-patient variability, varying dead time and disturbances are used to assess robustness of the controller. Furthermore, the controller guarantees stability under the operation conditions. Simulation results illustrate the use of the proposed controller. Copyright $\odot 2007$ IFAC
\end{abstract}

Keywords: Robustness, delay compensation, saturation, predictive control, drug dosing control, anesthesia.

\section{INTRODUCTION}

During the last decade, control technology has successfully influenced modern medicine through robotic surgery, electrophysiological system life support and image-guided therapy and surgery (Bailey and Haddad; 2005). Another area of medicine suited for applications of control is clinical pharmacology in general and a particular case is the anesthesia and critical care unit medicine. Within this particular group of applications, mon-

\footnotetext{
1 The authors gratefully acknowledge the contribution of Prof. Michel Struys from the Department of Anesthesia of Ghent University Hospital, and Tom De Smet from DEMED Company, Belgium. This work has been partially supported by the Latin American ALFA project II0385-FA (Process Systems Engineering). J.E. Normey-Rico gratefully acknowledges CAPES-BRASIL Contract BEXO 0828/05-0.
}

itoring and controlling the depth of anesthesia for patients during surgery offers interesting challenges to the modern engineer (Haddad et al.; 2003).

This topic captured the attention of engineers and clinicians already a decade ago (O'Hara et al.; 1992), starting with expert systems that offer advice to the anesthesiologist upon optimal drug infusion rate during clinical trials (Greenhow et al.; 1993). It soon became clear that control of anesthesia poses a manifold of challenges, with multivariable characteristics (Zbinden et al.; 1995), time delay between drug administration and the clinical effect which can lead to system oscillations (Bailey and Haddad; 2005), different dynamics depending on anesthetics substances (Absalom and Struys; 2005) and stability problems (Asbury; 1997). Further investigations proved Propofol to 
be an anesthetic tackled well in control problems (Kenny and Mantzaridis; 1999), while recent studies showed that the control performance may also depend on the controlled variable (Ting et al.; 2004).

Automatic feedback control of drug administration is ideally suited to anesthetized surgical patients since drugs with rapid onset times and short duration of action are frequently used (such as Propofol). The application of a closed-loop technology to drug delivery will assist physicians in avoiding both excessive over-dosages and underdosages in their patients. Ideally, a robust controller would then tackle over-dosing and underdosing by compensating for nonlinear drug responses, varying time delay, as well as inter- and intra-patient variation. Therefore, from control engineering standpoint, Model Predictive Control (MPC) plays a crucial role in solving such complex problems.

In this work, a predictive control strategy based on Smith predictor (Normey-Rico and Camacho; 2007), is applied to control the depth of anesthesia with promising results. In addition, it is studied a simple way to prove the system stability and to improve robustness under input saturation for this application case. An overview of the models used for prediction and for control is given in the next section. The MPC algorithm is described in section $I I I$ and stability and robustness are analyzed in the $I V^{t h}$ section. At the end of the paper some simulation results and the conclusions are presented.

\section{A REALISTIC PATIENT MODEL}

During anesthesia, when predictive control is intended for a precise administration of drugs, the model used in prediction becomes of vital importance. Such model must capture well-enough the dynamics of the patient in response to the specific drug considered (in our case Propofol). The relationship between drug infusion rate and the desired drug effect can be described with pharmacokinetic and pharmacodynamic models. Pharmacokinetic models describe the distribution of the drugs in the body and pharmacodynamic models describe the relationship between blood concentration of a drug and its clinical effect. Normally, these models can be identified for different kind of drugs by using a family of patients. For most anesthetic agents exist already several models (Absalom and Struys; 2005). When considering Propofol as the manipulated drug, the pharmacokinetics can be described by a 3-compartment model (Figure 1) (Absalom and Struys; 2005):

$$
\begin{aligned}
\dot{x}_{1}(t)= & -\left[k_{11}+k_{12}+k_{13}\right] x_{1}(t)+k_{21} x_{2}(t) \\
& +k_{31} x_{3}(t)+u(t), \\
\dot{x}_{2}(t)= & k_{12} x_{1}(t)-k_{21} x_{2}(t), \\
\dot{x}_{3}(t)= & k_{13} x_{1}(t)-k_{31} x_{3}(t),
\end{aligned}
$$

where $x_{1}$ (or $C_{p}$ ) denotes the concentration of drug in the central compartment (blood). The peripheral compartments $x_{2}$ and $x_{3}$ model the drug exchange of the blood with well and poorly perfused body times. The constants $k_{j i}$ for $i \neq j$, denote the transfer rate of drug from $j^{\text {th }}$ compartment to the $i^{\text {th }}$ compartment. The constant $k_{11}$ is the rate of drug metabolism and $u(t)$ is the infusion rate of the anesthetic drug (Propofol) into the central compartment (blood). The pharmacodynamics are characterized by a firstorder function related to the central compartment concentration $C_{p}$ in blood:

$$
\dot{C}_{e}(t)=-k_{e} C_{e}(t)+k_{e} C_{p}(t)
$$

where $k_{e}$ is a constant and $C_{e}$ is called effect site concentration.

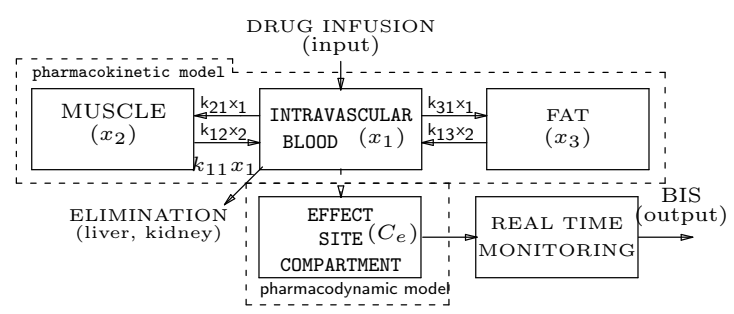

Fig. 1. Compartmental model.

For quantifying $C_{e}$ during real time monitoring, some commercial devices can be used. A recent device used by clinicians gives the Bispectral Index (BIS) that can be used as a measure of the depth of anethesia. The BIS is computed based on the electrical activity measure of brain, obtained through an Electroencephalograph. The BIS can vary from 0 to 100 and the device needs 15-30 seconds to process the data. Zero means that the patient does not have cerebral activity (dead) and 100 denotes that the patient is fully awake and consciousness (e.g. for $B I S=70$, the patient is awake but not consciousness). When undergoing surgery, the desired BIS target is 50 and must remain between 40 and 60 given an adequate sedation for the anesthesiologist. The BIS variable can be related to drug effect concentration $C_{e}$ by the empirical static nonlinear relationship (Bailey and Haddad; 2005), called also the Sigmoid curve:

$$
\operatorname{BIS}(t)=\left(\mathrm{E}_{0}-\mathrm{E}_{\max } \frac{C_{\mathrm{e}}(t-l)^{\gamma}}{C_{\mathrm{e}}(t-l)^{\gamma}+\mathrm{C}_{50}^{\gamma}}\right)
$$

where $E_{0}$ denotes the baseline (state without drug) value, which, by convention, is typically assigned a value of $100 \% ; E_{0}-E_{\max }$ denotes the minimum achievable BIS; $C_{50}$ is the drug concentration at half maximal effect and represents the patient's sensitivity to the drug; $l$ is the real time monitoring delay; and $\gamma$ determines the steepness of the curve in (3).

The constants present in the 3-compartment model (1) and pharmacodynamic model (2) are 
computed using the following equations for the Propofol drug (Absalom and Struys; 2005):

$$
\begin{aligned}
V_{c}= & 4.27, V_{2}=18.9-0.39(\text { age }-53), V_{3}=238, k_{e}=0.46 \\
k_{12}= & \frac{C_{l 2}}{V_{c}}, k_{13}=\frac{C_{l 3}}{V_{c}}, k_{21}=\frac{C_{l 2}}{V_{2}}, k_{31}=\frac{C_{l 3}}{V_{3}} \\
k_{11}= & \frac{C_{l 1}}{V_{c}}, C_{l 3}=0.836, C_{l 2}=1.29-0.024(\text { age }-53), \\
C_{l 1}= & 0.0456(\text { weight }-77)-0.0681(\mathrm{lbm}-59) \\
& 0.0264(\text { height }-177)+1.89,
\end{aligned}
$$

with $\mathrm{LBM}_{\mathrm{MALE}}: \mathrm{lbm}=1.1$ weight $-128 \frac{\text { weight }^{2}}{\text { height }^{2}}$, $\mathrm{LBM}_{\text {FEMALE }}: \mathrm{lbm}=1.07$ weight $-148 \frac{\text { weight }^{2}}{\text { height }^{2}}$, where the units of the weight, height and age are kilograms, centimeters and years respectively.

\section{APPROACH TO MODEL PREDICTIVE CONTROL}

Generally, an MPC algorithm consists of applying a control sequence that minimizes a multistage cost function $(J)$ of the form:

$$
\sum_{k=N_{1}}^{N_{2}}[y(t+k \mid t)-w(t+k)]^{2}+\sum_{k=0}^{N_{u}-1} \lambda[\Delta u(t+k \mid t)]^{2}
$$

subject to:

$$
\begin{gathered}
\Delta u_{\min } \leq \Delta u(t+k \mid t) \leq \Delta u_{\max } \quad \forall k=0, \ldots, N_{u}-1 \\
u_{\min } \leq u(t+k \mid t) \leq u_{\max } \quad \forall k=0, \ldots, N_{u}-1, \\
y_{\text {min }} \leq y(t+k \mid t) \leq y_{\max } \quad \forall k=N_{1}, \ldots, N_{2},
\end{gathered}
$$

where $N_{1}$ and $N_{2}$ are the minimum and maximum costing horizon, $N_{u}$ is the control horizon, $\lambda$ is the control weight, $w(t+k)$ is a future set-point or reference sequence, $\Delta u(t)$ is the incremental control action $\left(\Delta=1-q^{-1}\right.$; with $q^{-1}$ the backward shift operator) and $y(t+k \mid t)$ is the optimum $k$-step ahead prediction of the system output $y(t)$ on data up to time $t$.

\subsection{Computing the predictions}

The process output can be represented as:

$$
y(t)=x(t)+n(t)
$$

where: $x(t)$ is the model output when a control input $u(t)$ is applied and $n(t)$ represents the effect of the disturbances and modelling errors.

In the linear case, the model output $x(t)$ is given by the generic dynamic system model:

$$
x(t)=\left(B\left(q^{-1}\right) / A\left(q^{-1}\right)\right) u(t-d-1)
$$

where $d$ is the discrete time delay. The disturbance $n(t)$ can be modeled by:

$$
n(t)=C\left(q^{-1}\right) / D\left(q^{-1}\right) e(t),
$$

where $e(t)$ is uncorrelated (white) noise with zero mean value. $A\left(q^{-1}\right), B\left(q^{-1}\right), C\left(q^{-1}\right)$ and $D\left(q^{-1}\right)$ are polynomials.

The fundamental step in MPC methodology consists in predicting the process output at time instant $t$, indicated by $\{y(t+k \mid t), k=$ $\left.N_{1} \ldots N_{2}\right\}$, over the prediction horizon. This prediction is based on: (a) the measurements available at sampling time instant $t:\{y(t), y(t-$
$1), \cdots, u(t-1), u(t-2), \cdots\}$; and (b) the future values of the input signal, calculated at time $t$ : $\{u(t \mid t), u(t+1 \mid t), \cdots\}$.

Using the generic process model (5), the predicted values of the output are:

$$
y(t+k \mid t)=x(t+k \mid t)+n(t+k \mid t) .
$$

The prediction of $x(t+k \mid t)$ and $n(t+k \mid t)$ can be done respectively by recursion of the process model, by using filtering techniques, or based on the diophantine equations (De Keyser; 2003; Camacho and Bordons; 2004).

\subsection{Nonlinearity Compensation}

The nonlinearity introduced by the BIS variable is compensated by using the inverse of the nominal Sigmoid curve (3). As a result, a linear model for the MPC controller is obtained (Figure 2). The error on the nonlinearity compensation is assumed as an uncertainty on the linear model gain.

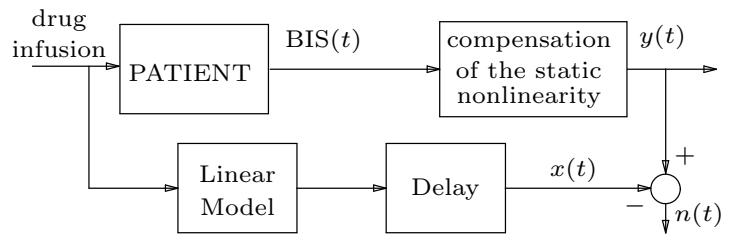

Fig. 2. Linear Structure.

In the prediction model, the controller uses the nominal Sigmoid curve for compensating the nonlinearity, independent on the patients which are tested in simulation. This assumes therefore that the controller must be robust enough to be able to compensate the inter-patient variability. Notice that external disturbances like surgical stimulation and blood loss are also present.

\subsection{Optimization Procedure}

The optimization procedure is a crucial step in MPC algorithms. The numerical complexity depends on the characteristics of the models in terms of linearity, constraints, number of manipulated and controlled variables, etc. For linear models without constraints there are some MPC techniques that solve the optimization procedure analytically (De Keyser; 2003). Moreover for deadtime processes the solution can be reduced to a linear controller of two degree of freedom plus a predictor (Normey-Rico and Camacho; 2007) and it is important when analysis is performed. For nonlinear and linear models with constraints, it is not possible to find an analytical solution. However, there are several powerful optimization methods to solve the optimization problem using iterative procedures (Gauss-Newton and LevenbergMarquardt methods or Sequential Quadratic Programming) (Biegler; 1998).

Either analytically, either by using iterative procedures, the optimal input sequence can be computed along the input horizon $N_{u}$. Nevertheless, 
due to the receding strategy of MPC, only the optimal input $u(t)$ at the present moment is applied to the process and for the next sample period the optimization problem is solved again.

From controller analysis standpoint an analytical solution is important. In the linear unconstrained case, the solution can be written as (Camacho and Bordons; 2004):

$$
\Delta u(t)=\mathbf{k}(\mathbf{w}-\mathbf{f}),
$$

where $\mathbf{k}$ is a constant matrix with dimension $1 \times$ $N$ (where $N=N_{2}-N_{1}+1$ ), w is a vector which contains the future reference, $\mathbf{f}=[f(t+$ $\left.\left.N_{1} \mid t\right), \ldots, f\left(t+N_{2} \mid t\right)\right]^{T}$ (or free response) is a vector which contains the output prediction for an input $u(t+k \mid t)=u(k-1), \forall k=0, \ldots, N-1$. Based on diophantine equations, the input can be expressed as (Normey-Rico and Camacho; 2007):

$$
\Delta u(t)=\mathbf{k w}-\frac{F^{B}\left(z^{-1}\right)}{B\left(z^{-1}\right)}[x(t+d)+R(z)(y(t)-x(t))],
$$

where $R(z)=\frac{B\left(z^{-1}\right) F^{C}\left(z^{-1}\right)}{F^{B}\left(z^{-1}\right) C\left(z^{-1}\right)}, \quad F^{B}\left(z^{-1}\right)$ and $F^{C}\left(z^{-1}\right)$ are polynomials which depend on the predictor model.

\subsection{Robust Model Predictive Controler (RMPC)}

As has been demonstrated by Normey-Rico and Camacho (2007) $R(z)$ can be used as a tuning parameter if an appropriate predictor structure is considered. In the nominal case, the tuning of $R(z)$ does not affect the set-point performance and only the disturbance rejection. To improve the robustness $R(z)$ must be a low pass filter. The tuning is performed using the denominator of $R(z)$ to achieve the desired low pass characteristics and the numerator of $R(z)$ to improve the closed loop response characteristics. In this application a second order filter of the form:

$$
R(z)=\frac{(1-\alpha)^{2}}{(1-\beta)} \frac{\left(1-\beta z^{-1}\right)}{\left(1-\alpha z^{-1}\right)^{2}}
$$

is used, where $\beta$ is chosen to eliminate the undesired root of $B\left(z^{-1}\right)$ and $\alpha$ is a tuning parameter chosen for a desired trade off between the robustness and disturbance rejection.

\section{ROBUSTNESS AND STABILITY ANALYSIS}

Several approaches on stability and robustness analysis can be found in literature for MPC controllers. However, the analysis is not so trivial when constraints apply or nonlinear models are used.

In this application, a specific case with constraints on the input $(u \geq 0)$ is studied, for the control horizon $N_{u}=1$ and the prediction horizons $N_{1}=1+d$ and $N_{2}=N+1+d$ where $N$ used as tuning parameter. Notice that for $N_{u}=1$, the constrained MPC controller is equivalent to clipping, a case valid only for monovariable systems (De Keyser; 2003). The term clipping assumes that the predictive controller does not take into account constraints while computing the optimal input rate to the process, but only afterwards, performing hard-limitations if constraints are violated.

To study the stability and robustness, the block diagram of Figure 3 is considered, since a linear MPC can be reduced in a block diagram containing a filtered Smith predictor for analysis purposes (Normey-Rico and Camacho; 1999). $H(z)=F^{B}\left(z^{-1}\right) / B\left(z^{-1}\right), L(z)$ is the loop transfer function of the system, $k_{w}=\sum_{i=1}^{N} \mathbf{k}(i)$, that is, the reference is considered $w(t+k)=w(t)$ and equal to the current set-point $r(t)$. Additionally there is a saturation nonlinearity at the input of the process (Figure 3). For stability analysis, the nonlinear element can be approximated by a describing function $(\phi)$. It is valid for most of the processes with low pass filter characteristics as is the case of the patient models.

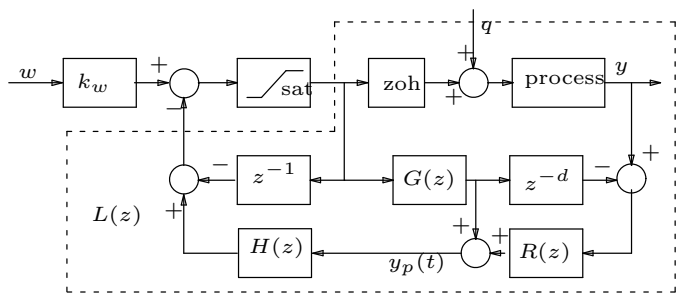

Fig. 3. Block diagram control scheme of the MPC.

For the case of saturation nonlinearity, the describing function is:

$$
\phi=\frac{2}{\pi}\left[\sin ^{-1}\left(\frac{s a t}{X}\right)+\frac{s a t}{X} \sqrt{1-\left(\frac{s a t}{X}\right)^{2}}\right],
$$

where sat is the amplitude of the saturation and $X$ the amplitude of a wave input of the form $X \sin (\omega t)$.

From Figure 3, the closed-loop frequency response is: $\frac{y}{w^{\prime}}=\frac{\phi L(j \omega)}{1+\phi L(j \omega)}$, where the characteristic equation is $1+\phi L(j \omega)=0$ or $L(j \omega)=-1 / \phi$.

When $L$ is minimum phase (as is the case for this drug dosing control application), according to the stability criteria, if the locus of $L(j \omega)$ does not intersect the locus of $-1 / \phi$, then the closed loop system is stable. Otherwise the system is unstable or presents a limit-cycle.

Figure 4 shows the locus of $-1 / \phi, L(j \omega)$ or Nyquist plot in the nominal case (continuous line) and when $\pm 10 \mathrm{~s}$ delay and $\pm 50 \%$ gain uncertainties are considered (dotted lines) for the set of patients and MPC controller from section 5. The locus of $-1 / \phi$ is plotted for the case of saturation 
nonlinearity, it starts at -1 and goes to $-\infty$. There is a critical point at -1 that must be considered for stability analysis. Notice that if the locus of $L(j \omega)$ does not encircle the critical point then it will not intersect $-1 / \phi$. Thus, if the closed-loop system without input saturation is stable, then it will remain stable under input saturation.

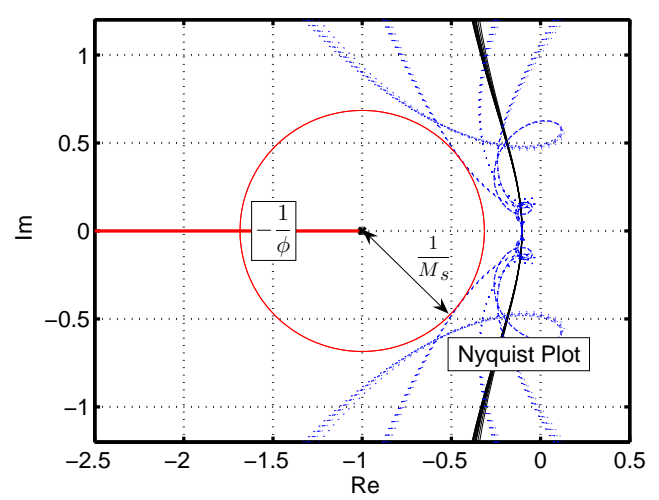

Fig. 4. Nyquist Plot considering the MPC controller, gain uncertainties and $\pm 10 s$ varying time delay.

In the case of modelling uncertainties, maximum and minimum limits on the parameter uncertainties must be considered for which the system must remain stable. That is, the locus of $L(j \omega)$ must not encircle -1 , for the system to be robustly stable. One way to define the robustness is through the maximum sensitivity $\left(M_{s}\right), M_{s}=\max _{\omega} \mid 1+$ $\left.L(j \omega)\right|^{-1}$, where $M_{s}$ can vary from 1 to $\infty$ and values between 1.5 and 2.5 are normally used (Normey-Rico and Camacho; 2007). Lower values of $M_{s}$ give higher robustness. For the studied case, using the Nyquist criteria, also it is possible to find the following sufficient stability condition of the system:

$$
\left|\Delta P\left(e^{j \omega}\right)\right|<\frac{\left|G\left(e^{j \omega}\right) H\left(e^{j \omega}\right)+1-e^{-j \omega}\right|}{\left|H\left(e^{j \omega}\right) R\left(e^{j \omega}\right)\right|},
$$

where $\Delta P$ is the additive modelling uncertainty. Notice that the low pass filter $R$ increases the right side term of (13). This confirms that the robustness of the proposed controller is improved compared to a standard MPC for time delay systems.

\section{RESULTS}

In this section, a set of 12 patient-models are studied and robustness of the controller analyzed.

\subsection{Patients}

The characteristics of these patients are given in Table 1. The MPC controller design parameters are chosen as: $N_{u}=1, N_{1}=6, N_{2}=16$ and the sample time $T_{s}=5 \mathrm{~s}$. Additionally for the RMPC controller are chosen the filter parameters

\begin{tabular}{|c|c|c|c|c|c|r|r|r|}
\hline $\mathrm{P}$ & Age & $\mathrm{L}$ & $\mathrm{W}$ & $\mathrm{G}$ & $C_{50}$ & $E_{0}$ & $E_{\max }$ & $\gamma$ \\
\hline 1 & 40 & 163 & 54 & $\mathrm{~F}$ & 6.33 & 98.80 & 94.10 & 2.24 \\
2 & 36 & 163 & 50 & $\mathrm{~F}$ & 6.76 & 98.60 & 86.00 & 4.29 \\
3 & 28 & 164 & 52 & $\mathrm{~F}$ & 8.44 & 91.20 & 80.70 & 4.1 \\
4 & 50 & 163 & 83 & $\mathrm{~F}$ & 6.44 & 95.90 & 102.00 & 2.18 \\
5 & 28 & 164 & 60 & $\mathrm{M}$ & 4.93 & 94.70 & 85.30 & 2.46 \\
6 & 43 & 163 & 59 & $\mathrm{~F}$ & 12.1 & 90.20 & 147.00 & 2.42 \\
7 & 37 & 187 & 75 & $\mathrm{M}$ & 8.02 & 92.00 & 104.00 & 2.10 \\
8 & 38 & 174 & 80 & $\mathrm{~F}$ & 6.56 & 95.50 & 76.40 & 4.12 \\
9 & 41 & 170 & 70 & $\mathrm{~F}$ & 6.15 & 89.20 & 63.80 & 6.89 \\
10 & 37 & 167 & 58 & $\mathrm{~F}$ & 13.7 & 83.10 & 151.00 & 1.65 \\
11 & 42 & 179 & 78 & $\mathrm{M}$ & 4.82 & 91.80 & 77.90 & 1.85 \\
12 & 34 & 172 & 58 & $\mathrm{~F}$ & 4.95 & 96.20 & 90.80 & 1.84 \\
\hline
\end{tabular}

Table 1. Patients characteristics $(\mathrm{P}=$ patient, $\mathrm{L}=$ length, $\mathrm{W}=$ weight and $\mathrm{G}=$ gender).

$\alpha=0.87$ and $\beta=-0.96$. For control purposes a maximum identification error of $10 \%$ is considered in the parameters Length and Weight, whereas the Age of the patient is considered to be well identified. These three parameters define the dynamic part of the model. The nominal Sigmoid curve parameters are chosen: $C_{50}=7.43, \gamma=3.01$, $E_{0}=100, E_{\max }=100\left(C_{50}\right.$ and $\gamma$ as the mean values of the patients). Additionally, a delay of $25 s$ is considered with $\pm 10 s$ of uncertainty (also a hypothetical case with $\pm 25 \mathrm{~s}$ of uncertainty is studied) between the effect site concentration and BIS measure. The variability of the Sigmoid curve between the modelled patients and the prediction model in the controller can be seen as a variation in the gain. In the operating region (BIS: $[40,60]$ ) the gain varies from $1.5 K$ to $0.5 K$, where $K$ is the gain of the model.

In order to ensure for all the patients a stable closed-loop response, the controller must be robust enough to guarantee the stability under identification parameters error and gain variability.

For the set of patients considered in this investigation, Table 2 shows the maximum sensitivity for the previously specified uncertainties using MPC and RMPC controllers. As expected the RMPC has better robustness than the MPC, specially for bigger uncertainties on the delay estimation.

\begin{tabular}{|l|c|c|c|c|}
\hline & $\mathrm{MPC} \pm 10 s$ & $\mathrm{RMPC} \pm 10 \mathrm{~s}$ & $\mathrm{MPC} \pm 25 s$ & $\mathrm{RMPC} \pm 25 s$ \\
\hline$M_{s}$ & 1.47 & 1.2 & 4 & 1.3 \\
\hline
\end{tabular}

Table 2. Maximum sensitivity considering $\pm 10 s$ and $\pm 25 s$ delay uncertainty, gain, length and weight uncertainties.

\subsection{Closed-loop simulations}

To enhance the advantages of using the RMPC over the classical control MPC technique, a comparative study is performed in simulations. Figures 5 and 6 show the closed-loop simulations for the controlled output (BIS). It is assumed that the model delay is $25 \mathrm{~s}$ and the patients delay $35 \mathrm{~s}$ $(+10 s$ error). The performance over the family of patients is affected due to the delay error and due to inter-patient variability, when using a nominal model for prediction in the controller strategies. The settling time has been defined here as the 

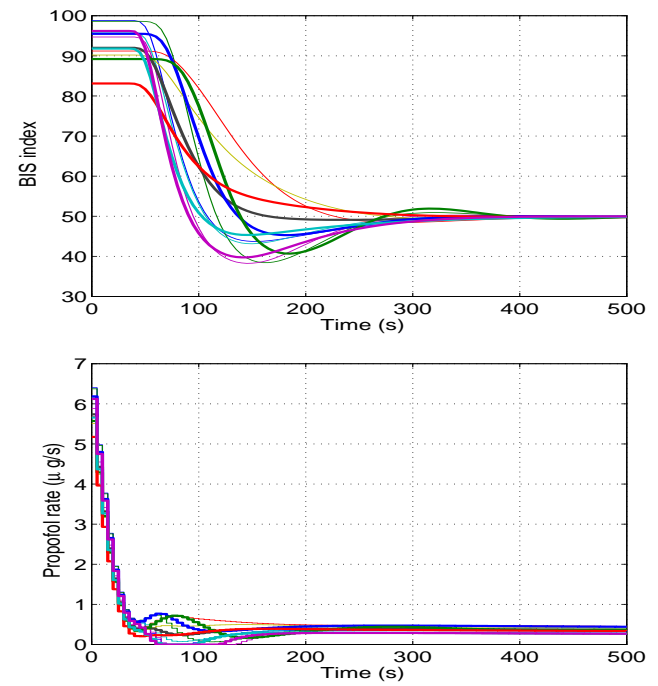

Fig. 5. Closed loop response for RMPC strategy.
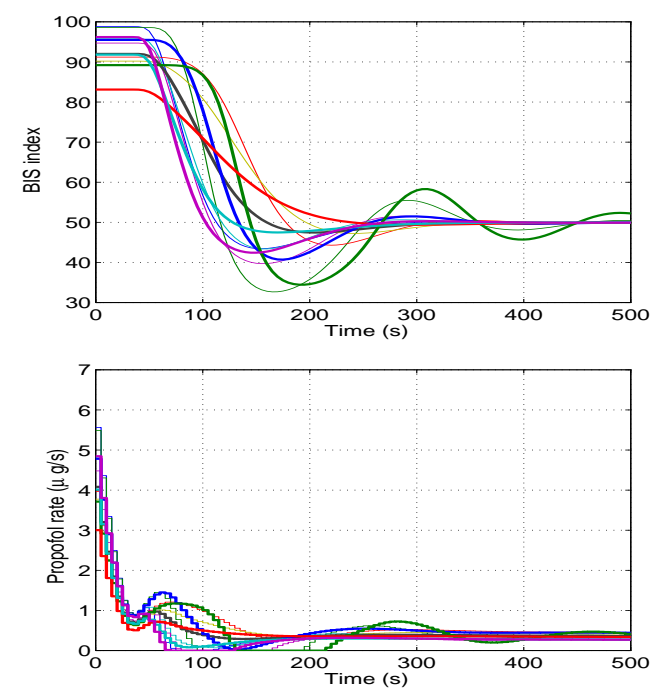

Fig. 6. Closed loop response for MPC strategy.

time needed by the controller to reach the setpoint (50 BIS) and stay within the accepted BIS limits $(40 ; 60)$. It can be observed that the RMPC strategy needs $190 s$ and the MPC strategy $238 s$ to achieve the desired BIS and stay within the safety limits. The undershoot in the worst case for the RMPC is $23.5 \%$ and for the MPC $35 \%$ respectively. Finally, it can be observed that the closed-loop response variability has been reduced with the RMPC strategy.

\section{CONCLUSIONS}

In this contribution, a Robust Model Predictive Controler strategy which can tackle in a robust manner the depth of anesthesia for patients undergoing surgery has been described and tested. Due to the fact that there is variation of time delay and the nonlinearity of the system consists of a static nonlinear gain, the closed-loop stability is guaranteed for the range where the time delay and gain varies due to real time monitoring and inter-patient variability respectively.

As a general remark, the tuning rules of the controller are intuitive to attain some performance specifications and are therefore suitable for clinical use.

\section{REFERENCES}

Absalom, A. and M. Struys (2005). An overview of TCI \& TIVA. Academia press.

Asbury, A. J. (1997). Feedback control in anesthesia. International J of Clinical Monitoring and Computing 14, 1-10.

Bailey, J. M. and W. M. Haddad (2005). Drug dosing control in clinical pharmacology. IEEE Control Systems Magazine 25(2), 35-51.

Biegler, L. T. (1998). Advances in nonlinear programming concepts for process control. Journal of Process Control 8(5), 301-311.

Camacho, E. F. and C. Bordons (2004). Model Predictive Control. Springer. Berlin.

De Keyser, R. (2003). Model based predictive control. Invited Chapter in UNESCO Encyclopedia of Life Support Systems (EoLSS), article 6.43.16.1 p. 30.

Greenhow, S. G., D. A. Linkens and A. J. Asbury (1993). Pilot study of an expert system adviser for controlling general anesthesia. British $J$ of Anesthesia 71, 359-365.

Haddad, W. M., T. Hayakawa and J. M. Bailey (2003). Nonlinear adaptive control for intensive care unit sedation and operating room hypnosis. In: American Control Conference. Vol. 2. pp. 1808-1813.

Kenny, G. N. and H. Mantzaridis (1999). Closedloop control of propofol anesthesia. British $J$ of Anesthesia 83(2), 223-230.

Normey-Rico, J. E. and E. F. Camacho (1999). Robustness effects of a prefilter in smith predictor based generalised predictive controller. IEE Proceedings, Control Theory and Applications 146(2), 179-185.

Normey-Rico, J. E. and E. F. Camacho (2007). Control of Dead-time Processes. Springer. Berlin.

O'Hara, D., D. Bogen and A. Noordergraaf (1992). The use of computers for controlling the delivery of anesthesia. Anesthesiology 77, 563581.

Ting, C. H., R. H. Arnott, D. A. Linkens and A. Angel (2004). Migrating from targetcontrolled infusion to closed-loop control in general anesthesia. Computer methods and programs in biomedicine 75, 127-139.

Zbinden, A. M., P. Feigenwinter, S. Petersen-Felix and S. Hacisalihzade (1995). Arterial pressure control with isoflurane using fuzzy logic. British $J$ of Anesthesia 74, 66-72. 